\title{
CARACTÉRISATION DES VARIÉTÉS HOMOLOGIQUES À L'AIDE DES INVARIANTS D'HOMOLOGIE D'INTERSECTION
}

\author{
JEAN-PAUL BRASSELET \\ SiGmA, CIRM-Luminy \\ Case 916, 13288 Marseille Cedex 09, France \\ E-mail: jpb@cirm.univ-mrs.fr \\ KARL-HEINZ FIESELER et LUDGER KAUP \\ Fakultät für Mathematik, Universität Konstanz \\ Postfach 5560, 7750 Konstanz, Germany \\ E-mail: barthel@cauchy.mathe.uni-konstanz.de
}

La conférence de J. P. Brasselet au Symposium de Varsovie a eu pour thème les problèmes actuels de l'homologie d'intersection. Nous en présentons ici l'un des aspects, résultat d'un travail commun réalisé dans le cadre du programme Procope et pendant lequel le second auteur a été chercheur associé au CNRS.

1. Le problème. Dans toute la suite les homologies et cohomologies seront prises à supports localement fermés et à coefficients dans un anneau $\mathbf{R}$ principal, sans diviseur de zero. On renvoie à $[\mathrm{GoMcP}]$ et $[\mathrm{KaFi}]$ pour les définitions et notations utilisées.

Dans leur article sur l'homologie d'intersection, [GoMcP, §6.4], M. Goresky et R. MacPherson ont fait la conjecture suivante :

Conjecture. Soit $X$ une pseudovariété normale. Si $I_{\mathbf{p}} H_{*}(X) \rightarrow I_{\mathbf{q}} H_{*}(X)$ est un isomorphisme, pour toutes perversités $\mathbf{p}<\mathbf{q}$, alors $X$ est une variété homologique.

H. C. King [Ki], a donné un contre-exemple à cette conjecture et a montré qu'elle devient vraie si l'on permet des perversités plus générales que celles de M. Goresky et R. MacPherson, appelées "loose perversities".

Une autre approche consiste à montrer que la conjecture de M. Goresky et R. MacPherson est vraie pour les seules perversités portant leur nom mais en ajoutant une hypothèse supplémentaire. C'est par exemple le cas des pseudovariétés normales telles

1991 Mathematics Subject Classification: Primary 55N33; Secondary 57N65.

The paper is in final form and no version of it will be published elsewhere. 
que les voisinages tubulaires des strates n'ont pas de "monodromie homologique" (voir [BraSa]). Dans ce travail, nous utilisons les invariants de l'homologie d'intersection introduits dans $[\mathrm{KpFi}]$ et $[\mathrm{FiKp} \mathrm{1]}$ pour donner un critère local pour lequel la conjecture est vraie.

Soit $X$ une pseudovariété normale de dimension réelle $n$. Nous noterons $\mathcal{P}_{\mathbf{p}}^{\bullet}$ le complexe de Deligne, à coefficients dans $\mathbf{R}$ et correspondant à la perversité (ou tolérance) $\mathbf{p}$.

De façon à comparer l'homologie d'intersection pour les perversités $\mathbf{p}$ et $\mathbf{q}$, on introduit le triangle distingué :

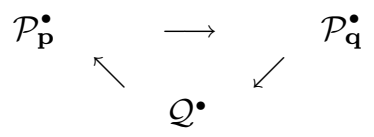

On en déduit une suite exacte longue de faisceaux dérivés

$$
\ldots \rightarrow \mathcal{H}^{j} \mathcal{P}_{\mathbf{p}}^{\bullet} \rightarrow \mathcal{H}^{j} \mathcal{P}_{\mathbf{q}}^{\bullet} \rightarrow \mathcal{H}^{j} \mathcal{Q}^{\bullet} \rightarrow \mathcal{H}^{j+1} \mathcal{P}_{\mathbf{p}}^{\bullet} \rightarrow \ldots
$$

et une suite exacte longue de modules d'hypercohomologie

$$
\ldots \rightarrow \mathbb{H}^{j}\left(X ; \mathcal{P}_{\mathbf{p}}^{\bullet}\right) \rightarrow \mathbb{H}^{j}\left(X ; \mathcal{P}_{\mathbf{q}}^{\bullet}\right) \rightarrow \mathbb{H}^{j}\left(X ; \mathcal{Q}^{\bullet}\right) \rightarrow \mathbb{H}^{j+1}\left(X ; \mathcal{P}_{\mathbf{p}}^{\bullet}\right) \rightarrow \ldots
$$

dans laquelle le module $\mathbb{H}^{j}\left(X ; \mathcal{P}_{\mathbf{p}}^{\bullet}\right)$ s'identifie à $I_{\mathbf{p}} H_{n-j}(X)$.

R e marque. Un isomorphisme $I_{\mathbf{p}} H_{j}(X) \rightarrow I_{\mathbf{q}} H_{j}(X)$, c'est à dire un isomorphisme $\mathbb{H}^{j}\left(X ; \mathcal{P}_{\mathbf{p}}^{\bullet}\right) \rightarrow \mathbb{H}^{j}\left(X ; \mathcal{P}_{\mathbf{q}}^{\bullet}\right)$ n'implique pas un isomorphisme de faisceaux $\mathcal{H}^{j} \mathcal{P}_{\mathbf{p}}^{\bullet} \rightarrow \mathcal{H}^{j} \mathcal{P}_{\mathbf{q}}^{\bullet}$.

Ainsi, si l'on cherche un critère global de caractérisation des variétés homologiques, King montre que l'on doit utiliser les "loose perversities". Dans ce qui suit, on donne un critère local, à l'aide des seules perversités de Goresky et MacPherson.

2. Rappels sur les invariants de l'homologie d'intersection. Etant donnée une filtration de $X$ :

$$
X=X_{n} \supset \Sigma=X_{n-2} \supset \cdots \supset X_{n-k} \supset \cdots \supset X_{0} \supset X_{-1}=\emptyset
$$

on note $S_{n-k}=X_{n-k} \backslash X_{n-k-1}$ et $U_{k}=X \backslash X_{n-k}=U_{k-1} \cup S_{n-(k-1)}$.

DÉfinition [KaFi, $§ 3.1]$. Soit p une perversité, on pose pour chaque ouvert $U \subset X$ :

$$
a_{U}(\mathbf{p}):=\sup \left\{i \in \mathbf{N} \cup\{\infty\}:\left.\mathcal{H}^{j}\left(\mathcal{P}_{\mathbf{p}}^{\bullet}\right)\right|_{U}=0,1 \leq j \leq i\right\}
$$

et $a(\mathbf{p}):=a_{X}(\mathbf{p})$.

Remarque. Si on a deux perversités $\mathbf{p}$ et $\mathbf{q}$ telles que $\mathbf{p} \leq \mathbf{q}$, alors $a(\mathbf{p}) \geq a(\mathbf{q})$.

R e m arque. $X$ est une variété homologique si et seulement si on a $a(\mathbf{p})=\infty$ pour toute perversité $\mathbf{p}$.

DÉfinition [KaFi, §3.3]. Soit $\mathbf{p}$ une perversité, on pose :

$$
k(\mathbf{p}):=\min \left\{i \in \mathbf{N} \cup\{\infty\}:\left.\widetilde{\mathcal{H}} \bullet\left(\mathcal{P}_{\mathbf{p}}^{\bullet}\right)\right|_{S_{n-i}} \neq 0\right\}
$$

où $\widetilde{\mathcal{H}} \bullet\left(\mathcal{P}_{\mathbf{p}}^{\bullet}\right)=\mathcal{H}^{\bullet}\left(\tau^{\geq 1} \mathcal{P}_{\mathbf{p}}^{\bullet}\right)$.

Remarque. Si on a deux perversités $\mathbf{p}$ et $\mathbf{q}$ telles que $\mathbf{p} \leq \mathbf{q}$, alors $k(\mathbf{p}) \geq k(\mathbf{q})$.

DÉfinition $[\mathrm{KaFi}, \S 6]$. On dit que la pseudovariété $X$ est exceptionnelle ou de type $\mathbf{E}$ (exceptionnel par rapport à $\mathbf{R}$ ) si et seulement si : 
1) $k=k(\mathbf{t})$ est pair,

2) $a_{U_{k+1}}(\mathbf{t})=\frac{k}{2}-1$.

Remarquons qu'une pseudovariété exceptionnelle $X$ satisfait :

$$
\left.\mathcal{H}^{j}\left(\mathcal{P}_{\mathbf{t}}^{\bullet}\right)\right|_{S_{n-k}}= \begin{cases}0 & \text { si } j \neq k / 2, \\ \text { est de torsion et non nul } & \text { si } j=k / 2 .\end{cases}
$$

En effet, [KaFi, §5.22] implique, pour $k=k(\mathbf{t})$,

$$
\widetilde{\mathcal{H}}^{j} \mathcal{P}_{\mathbf{t}, X}^{\bullet} \cong \operatorname{Ext}\left(\mathcal{H}^{k-j} \mathcal{P}_{\mathbf{t}, X}^{\bullet} ; \mathbf{R}\right) \oplus \operatorname{Hom}\left(\mathcal{H}^{k-j-1} \mathcal{P}_{\mathbf{t}, X}^{\bullet} ; \mathbf{R}\right)
$$

pour $x \in S_{n-k}$ et $j \leq k-2$. Par conséquent, $\mathcal{H}^{k / 2} \mathcal{P}_{\mathbf{t}, x}^{\bullet}$ est de torsion et non nul, puisque $a_{U_{k+1}}(\mathbf{t})=\frac{k}{2}-1$.

R e m a r qu e. Il n'existe évidemment pas de pseudovariété exceptionnelle par rapport à un corps $\mathbf{K}$. Si l'anneau $\mathbf{R}$ contient un sous-corps $\mathbf{K}$, en particulier si la caractéristique de $\mathbf{R}$ est non nulle, alors l'homologie d'intersection $I_{\mathbf{p}} H_{\bullet}(X ; \mathbf{R})$, de même que l'homologie locale $\mathcal{H}_{\bullet}^{\mathbf{R}}$ satisfont à la formule covariante des coefficients universels, $\mathbf{R}$ étant considéré comme espace vectoriel $\mathbf{K}$. Dans ce cas, $X$ est une variété homologique par rapport à $\mathbf{R}$ si et seulement s'il en est ainsi par rapport à $\mathbf{K}$. On pourrait alors, en cherchant les variétés homologiques, ne pas considérer les espaces de type $\mathbf{E}$.

D'autre part, si $X$ est une variété homologique par rapport à $\mathbb{Z}_{p}$, pour tout $p$ premier, alors $X$ est une variété homologique par rapport à $\mathbb{Z}$, puisque l'homologie locale satisfait la formule des coefficients universels, même si $\mathbf{R}$ n'est pas un corps.

Proposition [KaFi, §6.2]. Si $X$ n'est pas de type $\mathbf{E}$, et si $\mathbf{p}$ est une perversité satisfaisant $\mathbf{p}(k(\mathbf{t})) \geq \mathbf{n}(k(\mathbf{t}))$, où $\mathbf{n}$ est la perversité moitié supérieure, alors on a $a(\mathbf{p})=a(\mathbf{t})$.

Corollaire. Si X n'est pas de type $\mathbf{E}$, et si la perversité $\mathbf{p}$ satisfait l'inégalité $\mathbf{p}(k(\mathbf{p})) \geq \mathbf{n}(k(\mathbf{p}))$, alors on $a$ :

$$
2 a_{U_{k+1}}(\mathbf{p})+3 \leq k(\mathbf{p}) .
$$

La démonstration du corollaire utilise celle de la proposition (cf. [KaFi, §6.2]).

Remarque. Si on a une stratification d'une variété complexe (en fait, si on a une stratification en strates de codimensions paires), on peut remplacer, dans la formule du corollaire, "+3" par "+4".

Proposition [KaFi, §6.1]. Si $X$ n'est pas de type $\mathbf{E}$, alors il vient : $k(\mathbf{t})=\min (k(\mathbf{p})$, $k(\mathbf{t}-\mathbf{p}))$.

\section{Résultats et conséquences sur les variétés homologiques}

RÉSultat 1. X est une variété homologique si et seulement si $X$ n'est pas de type $\mathbf{E}$ et il existe une perversité $\mathbf{p}$ telle que, en posant $k:=k(\mathbf{p})$, on a $\mathbf{p}(k) \geq\left[\frac{k-1}{2}\right]$ et $2 a_{U_{k+1}}(\mathbf{p})+2 \geq k$.

RÉSULTAT 2. X est une variété homologique si et seulement si il existe une perversité $\mathbf{p}$ telle que, si $k:=k(\mathbf{p})$, alors $\mathbf{p}(k) \geq\left[\frac{k}{2}\right]$ et $2 a_{U_{k+1}}(\mathbf{p})+1 \geq k$.

Comme on a toujours l'inégalité $a(\mathbf{p}) \leq a_{U_{k+1}}(\mathbf{p})$, les résultats 1 et 2 entraînent : 
RÉSultat 3. $X$ est une variété homologique si et seulement si $X$ n'est pas de type $\mathbf{E}$ et s'il existe une perversité $\mathbf{p} \geq \mathbf{n}$ telle que $2 a(\mathbf{p})+2 \geq n$.

RÉSULTAT 4. $X$ est une variété homologique si et seulement s'il existe une perversité $\mathbf{p} \geq \mathbf{m}+\mathbf{1}$ telle que $2 a(\mathbf{p})+1 \geq n$.

Rem arque. Si $k$ est pair, on peut remplacer " $+2 "$ par " $+3 "$ dans le résultat 1 . De même dans le résultat 3 , si les strates de la stratification de $X$ sont de codimensions paires. En effet, si $k$ est pair, la remarque suivant le corollaire ci-dessus entraîne que $2 a_{U_{k+1}}(\mathbf{p})+4 \leq k$, donc $2 a_{U_{k+1}}(\mathbf{p})+3 \leq k$ implique $a_{U_{k+1}}(\mathbf{p})=\infty$.

Remarque. Si $k_{0}$ est la codimension maximale des strates, on peut remplacer $n$ par $k_{0}$ dans les énoncés 3 et 4 .

Exemple 1 (Cas où $n=3$ ). Dans ce cas, la perversité $\mathbf{n}=\mathbf{t}$ est la seule perversité différente de zero. Pour $X$ normale, le lieu singulier $\Sigma$ est de codimension $k=3$ ou est vide. Donc, $X$ est une variété homologique si et seulement si $a(\mathbf{n}) \neq 0$. Comme exemple singulier, on peut prendre le cône ouvert $\stackrel{c}{c}(L)$ sur une surface de Riemann compacte $L$ non rationnelle. Cette construction est à la base du contre-exemple de King (avec pour $L$, un tore).

Exemple 2 (Cas où $n=4$ ). Dans ce cas, nous obtenons les résultats suivants :

Si $k=3$, et si $\mathbf{p}(3) \geq 1$, alors, si $X$ n'est pas une variété homologique, $a(\mathbf{p})$ doit être 0 .

Si $k=4$, et si $X$ n'est pas de type $\mathbf{E}$, alors $X$ n'est pas une variété homologique si et seulement s'il existe une perversité $\mathbf{p}$ telle que $\mathbf{p}(4) \geq 1$ et $a(\mathbf{p})=0$.

Si $k=4$, et si $X$ est de type $\mathbf{E}$, alors $\left.\mathcal{H}^{1}\left(\mathcal{P}_{\mathbf{t}}^{\bullet}\right)\right|_{S_{0}}=0$ et $\left.\mathcal{H}^{2}\left(\mathcal{P}_{\mathbf{t}}^{\bullet}\right)\right|_{S_{0}} \neq 0, a(\mathbf{t})=1$.

R e marque. Jusqu'à présent, on a comparé les perversités $\mathbf{p}$ et $\mathbf{0}$. Il semble plus intéressant de comparer $\mathbf{p}$ avec des perversités moins distantes : Pour un espace complexe $X$, topologiquement localement intersection complète, on peut remplacer $a(\mathbf{p})=a(\mathbf{0}, \mathbf{p})$ par $a(\mathbf{q}, \mathbf{p})$, quel que soit $\mathbf{q}$ telle que $\mathbf{q}(k) \leq \mathbf{m}(k)-1$ pour $k \geq 2$. En utilisant [FiKp 1] et [FiKp 2], on a un résultat analogue dans le cas complexe général, moyennant l'invariant $\operatorname{tab}(X)$, qui mesure la "distance" à une intersection complète locale.

\section{Références}

[BraSa] J.-P. Brasselet et M. Saralegi, Variétés homologiques et homologie d'intersection, C. R. Acad. Sci. Paris Sér. I 314 (1992), 847-850.

[FiKp 1] K.-H. Fieseler and L. Kaup, The vanishing invariants in intersection homology of complex spaces, dans : Singularities, Banach Center Publ. 20, 1988, 225-235.

[FiKp 2] - - Q Quasi-isomorphic perversities and obstruction theory for pseudomanifolds, ibid., 199-223.

[GoMcP] M. Goresky and R. MacPherson, Intersection homology theory, Topology 19 (1980), 135-162.

[KaFi] L. Kaup and K.-H. Fieseler, Singular Poincaré duality and intersection homology, in: Proc. 1984 Vancouver Conf. in Algebraic Geometry, Amer. Math. Soc., Providence, 1986, 113-161.

[Ki] H. King, Intersection homology and homology manifolds, Topology 21 (1982), 229 234 . 